OPEN ACCESS

Edited by:

Yuka Kotozaki,

Iwate Medical University, Japan

Reviewed by:

Vu Duy Kien,

Independent Researcher,

Hanoi, Vietnam

Chen Zhang,

Shanghai Jiao Tong University, China

${ }^{*}$ Correspondence:

Peng Luo

luopeng@gmc.edu.cn

Xing Zhao

xingzhao@scu.edu.cn

tThese authors have contributed equally to this work and share first authorship

Specialty section:

This article was submitted to

Public Mental Health,

a section of the journal

Frontiers in Public Health

Received: 26 September 2021 Accepted: 22 November 2021

Published: 14 December 2021

Citation:

Liu Q, Wangqing P, Baima Y, Wang S, Shen Z, Zhou J, Song H, Liu Y, Liu X, Luo $P$ and Zhao $X$ (2021) Comorbid

Depressive and Anxiety Symptoms and Their Correlates Among 93,078 Multiethnic Adults in Southwest China.

Front. Public Health 9:783687. doi: 10.3389/fpubh.2021.783687

\section{Comorbid Depressive and Anxiety Symptoms and Their Correlates Among 93,078 Multiethnic Adults in Southwest China}

\author{
Qiaolan Liu ${ }^{1 \dagger}$, Pingcuo Wangqing ${ }^{2 \dagger}$, Yangji Baima ${ }^{3}$, Songmei Wang ${ }^{4}$, Zhuozhi Shen ${ }^{5}$, \\ Jing Zhou ${ }^{6}$, Huan Song ${ }^{7}$, Yuanyuan Liu ${ }^{1}$, Xiang Liu ${ }^{1}$, Peng Luo ${ }^{8 *}$ and Xing Zhao ${ }^{1 *}$ on \\ behalf of the China Multi-Ethnic Cohort (CMEC) Collaborative Group
}

\footnotetext{
${ }^{1}$ West China School of Public Health and West China Fourth Hospital, Sichuan University, Chengdu, China, ${ }^{2}$ Tibet Center for Disease Control and Prevention, Lhasa, China, ${ }^{3}$ Department of Preventive Medicine, College of Medicine, Tibet University, Lhasa, China, ${ }^{4}$ School of Public Health, Kunming Medical University, Kunming, China, ${ }^{5}$ Chongqing Municipal Center for Disease Control and Prevention, Chongqing, China, ${ }^{6}$ Chenghua District Center for Disease Control and Prevention, Chenghua, China, ${ }^{7}$ West China Biomedical Big Data Center of West China Hospital, Medical Big Data Center, Sichuan University, Chengdu, China, ${ }^{8}$ The Key Laboratory of Environmental Pollution Monitoring and Disease Control, School of Public Health, Ministry of Education, Guizhou Medical University, Guiyang, China
}

Background: Depressive symptoms and anxiety symptoms commonly coexist and severely increases the disease burden worldwide. Little is known about the patterns and correlates of comorbid depressive and anxiety symptoms among the multiethnic populations of China.

Methods: This population-based study investigated the comprehensive associations of comorbid depressive and anxiety symptoms with lifestyles, stressful life events, chronic diseases, and physical and mental well-being among 93,078 participants (37,193 men, 55,885 women) aged 30-79 years across seven ethnic groups in Southwest China. Multivariable logistic regression models were used to estimate associations.

Results: Overall, $2.9 \%(2.1 \%$ in men and $3.5 \%$ in women) participants had comorbid depressive and anxiety symptoms; there was considerable heterogeneity among multiethnic populations. Participants with chronic diseases were more likely to have comorbidity than those without them; people with rheumatic heart disease reported the highest risk, with an odds ratio (OR) of 6.25 and 95\% confidence interval $(\mathrm{Cl})$ of 4.06-9.62. Having experienced 3 or more stressful life events (OR, 8.43, 95\% Cl: 7.27-9.77), very poor self-rated health status (OR, 33.60, 95\% Cl: 25.16-44.87), and very unsatisfied life (OR, 33.30, 95\% Cl: 23.73-46.74) had strong positive associations with comorbid depressive symptoms and anxiety symptoms, with a dose-response relationship $(P<0.05)$. High frequency of physical activity had negative associations. All the associations were stronger than depressive symptoms alone or anxiety symptoms alone. 
Conclusions: Our findings emphasize the need to focus on the vulnerable ethnic groups with comorbid depressive and anxiety symptoms, ultimate for help early prevention and improvement of health equity in the underdevelopment and high urbanization areas.

Keywords: comorbid depressive and anxiety symptoms, multiethnic adult, chronic disease, physical and mental well-being, psychosocial factor

\section{INTRODUCTION}

Depression and anxiety are two highly prevalent and disabling mental disorders that commonly coexist $(1,2)$. Comorbid depression and anxiety can occur in individuals of all ages, seriously impairing social function, increasing psychosocial disability, and reducing the quality of life. Moreover, comorbid depression and anxiety are more likely to increase recurrence and lead to suicide; it is associated with an even higher risk of mortality than depression alone or anxiety alone. Globally over 264 million people of all ages suffer from depressive disorder; it is one of the top three leading causes of years lived with disability (YLDs) for both sexes combined in the world (3). The prevalence estimate for anxiety disorders of $7.3 \%$ from a systematic review for 44 countries constitutes a huge global burden of disease (4). Mental and substance use disorders account for $7.4 \%$ of all disability-adjusted life years (DALYs) worldwide, in which depressive disorder account for $40.5 \%$ and anxiety disorders account for $14.6 \%$ (5). Identifying and managing comorbidities is essential because depression and anxiety, even if they are independent, contribute to a significant proportion of the global burden of disease $(4,6,7)$.

Depressive symptoms and anxiety symptoms are reversible at early stages and can be treated with low-cost therapeutics $(8,9)$. Early interventions directly promote the treatment effects of other chronic diseases and reduce the burden of diseases, in turn leading to reduced health resource consumption (10). To achieve early management and intervention, early screening of comorbid depressive and anxiety symptoms warrants exploration.

Evidence on early screening of comorbid depressive and anxiety symptoms in mainland Chinese community populations is scarce. A limited number of studies are either based on elderly populations or in small sample community populations $(11,12)$, underscoring the importance of conducting studies in large general populations. Furthermore, research in multicultural vulnerable populations is limited, especially in China, where is economically underdeveloped yet has undergone rapid urbanization in recent years. Urbanizing leads to changes in the living environment and lifestyles, culture adjustment, job competition, life stress, and thus might cause mental health problems (13-15). The lack of scientific information on comorbid depressive and anxiety symptoms hinders policymakers from developing health services and allocating sufficient resources and intervention programs in these areas.

In this study, we examined the prevalence of comorbid depressive and anxiety symptoms among seven ethnic groups, including Tibetan, Miao, Bai, Bouyei, Dong, Yi, and Han in Southwest China using baseline data from the China multiethnic cohort (CMEC) (16). We also investigated the comprehensive factors that might influence the development of comorbid depressive and anxiety symptoms, including socioeconomic characteristics, lifestyles, stressful life events, sleep disorders, chronic diseases, and physical and mental well-being.

\section{MATERIALS AND METHODS}

\section{Study Participants}

Participants included in this study were from the baseline survey of CMEC conducted between May 2018 and September 2019 in Southwest China. The design and conduct of CMEC have been described elsewhere (16). Briefly, the CMEC was established in five provinces of Southwest China, including Tibet, Sichuan, Chongqing, Guizhou, and Yunan. In addition to the Han ethnic group, six ethnic minority community populations (Tibetan, Yi, Bai, Miao, Dong, and Bouyei) were included in consideration of both sex ratio and age ratio using a multistage, stratified cluster sampling method. The inclusion criteria and exclusion criteria were strictly executed in the process of the CMEC study. Overall, 99,556 participants aged 30-79 years (18-79 years in Tibetan sample) and of the characteristics of permanent residents were enrolled in the CMEC, including 55,443 Han, 12,730 Tibetan, 6,283 Yi, 6,310 Bai, 5,559 Miao, 7,239 Dong, and 5,992 Bouyei. The current study included 93,078 participants aged $30-79$ years (mean 51.8 years, SD 11.5 years) who were surveyed for both depressive symptoms and anxiety symptoms.

\section{Data Collection}

A tablet computer with a self-developed application (CMES App) that had an automatic audio recording function was used to collect the questionnaire information by a face-toface interview. The whole interview was implemented by welltrained interviewers who were medical workers from the local medical institutions or medical students from the local colleges, particularly familiar with the local ethnic language. The medical examinations provided information including height, weight, blood pressure, heart rate, ultrasound, X-rays, blood, and urine tests. Medical examinations and blood and urine collection strictly followed standard operating procedures (SOPs). The questionnaire covered socioeconomic status (age, gender, marital status, education, family income, occupation), lifestyle habits (smoking, alcohol drinking, tea consumption, dietary habits and physical activity), stressful life events, sleeping patterns, personal and family medical history, and psychological conditions. 


\section{Assessment of Depressive Symptoms and Anxiety Symptoms}

Depressive symptoms and anxiety symptoms were investigated using the PHQ-2 and GAD-2 questionnaires, respectively. These two questionnaires are two-item screening questionnaires based on the Diagnostic and Statistical Manual of Mental DisordersFourth Edition (DSM-IV) to assess depressive and anxiety symptoms in the past two weeks, respectively through the following two problems: (1) little interest or pleasure in doing things, and (2) feeling down, depressed, or hopeless (PHQ$2)$; (1) feeling nervous, anxiety, or on edge, and (2) not being able to stop or control worrying (GAD-2). For each item, the response options are "Not at all," "Several days," "More than half the days," and "Nearly every day," scored as $0,1,2$, and 3, respectively. The total score of both the PHQ-2 and GAD-2 ranges from 0 to 6 . A cutoff of 3 was adopted to identify depressive symptoms and anxiety symptoms. These two ultrashort screening tools considerably enhance the efficiency of screening for and monitoring depressive symptoms and anxiety symptoms in busy primary care practice and large populationbased epidemic surveys (17-21).

We conducted a repeated study with a sample of $10 \%$ of the total population from the baseline study from June to October 2020. The questionnaire survey, medical examination, and biometric sample collection were mostly the same as the baseline, but the PHQ-9, GAD-7 and a few other questions were added. The 8,229 participants of Han, Yi, Bai, and Miao nationality from $10 \%$ of the repeated study population were surveyed simultaneously on four scales of PHQ-9, PHQ-2, GAD-7, and GAD-2. PHQ-9 and GAD-7 classify diagnoses according to the Diagnostic and Statistical Manual of Mental Disorders fourth edition (DSM-IV) $(18,22)$. The Cronbach alpha coefficients were 0.73 for the PHQ-2 and 0.74 for the GAD-2 in this study. The correlation between the PHQ-9 and the PHQ2 was 0.813 . The Cronbach's alpha coefficients for the PHQ-9 and PHQ-2 were 0.809 and 0.691 , respectively. Using the PHQ9 with a cutoff of 10 as the threshold value, the area under the ROC curve for the PHQ-2 was 0.948 , and the specificity and sensitivity of the PHQ-2 were 98.2 and $64.6 \%$, respectively. The correlation between the GAD-7 and the GAD-2 was 0.906 in this same sample. The Cronbach's alpha coefficients of the GAD-9 and GAD-2 were 0.898 and 0.749 , respectively. Using GAD-7 with a cutoff of 10 as the threshold value, the area under the ROC curve for GAD-2 was 0.987 , and the specificity and sensitivity of GAD-2 were 98.2 and $92.9 \%$, respectively. All these results demonstrated good reliability and validity of the PHQ-2 and GAD-2 for our population sample.

\section{Assessment of Sleep Disorders}

Participants were asked whether they had any of the following three symptoms in at least three days or more in a week in the past month: (1) having trouble falling asleep after going to bed or waking up in the middle of the night; (2) waking up too early and not being able to get back to sleep; and (3) having trouble staying alert while at work, eating or meeting people during the daytime. Participants who answered "Yes" to any of the above three items were classified as having sleep disorders (23).

\section{Assessment of Stressful Life Events}

Participants were asked whether they had experienced the following 10 stressful events during the past two years: (1) divorce/separation; (2) loss of job/retirement; (3) business failure or bankruptcy; (4) being violently attacked/raped; (5) serious family internal contradictions and conflicts; (6) a serious injury or car accident; (7) seriously ill or death of a spouse; (8) seriously ill or death of other close family members; (9) serious natural disasters (e.g., drought or flood); (10) loss of the source of income/living in debt (24).

\section{Assessment of Life Satisfaction and Self-Rated Health Status}

One item was designed to ask participants whether they were satisfied with their life. The answer was grouped into five orderly categories as "very satisfied," "satisfied," "general," "not satisfied," and "not satisfied at all." Participants were also asked what they think of their current health status. The answer was also grouped into five orderly classes: "very good," "good," "medium," "poor," and "very poor" (24).

\section{Assessment of Common Chronic Diseases}

Information about the occurrence of cancers and the top 20 kinds of common chronic diseases was collected from the local disease surveillance system in the Chinese western multiple minority areas. The diseases included rheumatic health disease, coronary heart disease, diabetes, stroke, and some plateau-specific diseases; these diseases must be confirmed by a medical institution at or above the level of the county hospital before the baseline survey.

\section{Statistical Analysis}

The mean values and proportions with selected baseline characteristics were calculated by the presence of depressive symptoms, anxiety symptoms, and their comorbidity. To analyze changes of comorbidity of depressive and anxiety symptoms with age, we used the Chi-square trend test. To explore the potential influencing factors for depressive symptoms, anxiety symptoms, and their comorbidity, logistic regression models were used to calculate multivariable-adjusted ORs and 95\% CIs. All the ORs in the tables and figures were adjusted simultaneously for age grouped into five categories per 10 years: gender, education (illiteracy, primary, middle school, and college), family income $(<12,000,12,000-19,999,20,000-59,999,60,000-$ 99,999, 100,000-199,999, and $\geq 200,000 \mathrm{RMB} /$ year), occupation (employed, retirement, unemployment), and marriage (married, divorced, widowed, and single). The other variables included smoking (no, past, current), alcohol consumption (no, past, current), tea consumption (no, yes), BMI ( $<18.5,18.5-23.9$, $24-27.9$, and $\left.\geq 28 \mathrm{~kg} / \mathrm{m}^{2}\right)$, and physical activity $(<12.0,12.0-$ $37.3, \geq 37.3 \mathrm{MET}-\mathrm{h} /$ day). The frequency of tea consumption was classified into four categories: past, 1-2, 3-5, and 6-7 d/week. The duration of tea consumption was grouped into three categories according to three quantiles: $<15,15-39$, and $\geq 39$ years. The frequency and duration of alcohol consumption and the duration 
TABLE 1 | Sociodemographic characteristics and selected characteristics by mental health status.

\begin{tabular}{|c|c|c|c|c|c|}
\hline & Overall & $\begin{array}{l}\text { Depressive } \\
\text { symptoms }\end{array}$ & $\begin{array}{l}\text { Anxiety } \\
\text { symptoms }\end{array}$ & $\begin{array}{l}\text { Neither } \\
\text { depression nor } \\
\text { anxiety }\end{array}$ & $\begin{array}{l}\text { Comorbid } \\
\text { depressive and } \\
\text { anxiety } \\
\text { symptoms }\end{array}$ \\
\hline $\begin{array}{l}\text { Age }(x \pm S D \text {, } \\
\text { years })\end{array}$ & $51.8 \pm 11.5$ & $53.9 \pm 11.5$ & $54.1 \pm 11.0$ & $51.6 \pm 11.5$ & $54.1 \pm 11.1$ \\
\hline $40-$ & 28,265 & $1,341(4.7)$ & $1,574(5.6)$ & 26,098 & $748(2.7)$ \\
\hline $50-$ & 24,759 & 1,422 (5.7) & 1,823 (7.4) & 22,349 & 835 (3.4) \\
\hline $60-$ & 17,515 & 1,113 (6.5) & $1,314(7.5)$ & 15,704 & $616(3.5)$ \\
\hline $70-79$ & 6,806 & 475 (7.0) & $505(7.4)$ & 6,069 & 243 (3.6) \\
\hline \multicolumn{6}{|l|}{ Gender (\%) } \\
\hline Illiteracy & 23,277 & 1,937 (8.3) & 2,458 (10.6) & 20,079 & $1,197(5.1)$ \\
\hline Primary & 23,948 & 1,332 (5.6) & $1,560(6.5)$ & 21,788 & $732(3.1)$ \\
\hline Middle school & 35,477 & 1,376 (3.9) & $1,545(4.4)$ & 33,235 & $679(1.9)$ \\
\hline College & 10,375 & 302 (2.9) & 237 (2.3) & 9,949 & $113(1.1)$ \\
\hline \multicolumn{6}{|l|}{ Marriage (\%) } \\
\hline Married & 82,658 & 4,095 (4.9) & 4,871 (5.9) & 75,941 & $2,249(2.7)$ \\
\hline Divorce & 3,613 & $270(7.5)$ & $273(7.6)$ & 3,217 & $147(4.1)$ \\
\hline Widowed & 5,692 & $514(9.0)$ & 600 (10.5) & 4,880 & 302 (5.3) \\
\hline Single & 1,114 & $68(6.1)$ & $56(5.0)$ & 1,013 & $23(2.1)$ \\
\hline \multicolumn{6}{|l|}{ Occupation (\%) } \\
\hline $20,000-59,999$ & 33,325 & $1,579(4.7)$ & 1,867 (5.6) & 30,709 & $830(2.5)$ \\
\hline 60,000-99,999 & 13,997 & $481(3.4)$ & 539 (3.9) & 13,209 & $232(1.7)$ \\
\hline $100,000-199,999$ & 10,145 & 302 (3.0) & 305 (3.0) & 9,670 & $132(1.3)$ \\
\hline$\geq 200,000$ & 2,562 & $71(2.8)$ & $66(2.6)$ & 2,455 & $30(1.2)$ \\
\hline \multicolumn{6}{|l|}{ Nationality (\%) } \\
\hline Han & 55,281 & $2,448(4.4)$ & 2,415 (4.4) & 51,583 & $1,165(2.1)$ \\
\hline Dong & 7,222 & 764 (10.6) & $1,045(14.5)$ & 5,883 & $470(6.5)$ \\
\hline Bouyei & 5,954 & $400(6.7)$ & $641(10.8)$ & 5,154 & 241 (4.1) \\
\hline Yi & 6,276 & $406(6.5)$ & $565(9.0)$ & 5,574 & 269 (4.3) \\
\hline Miao & 5,538 & $633(11.4)$ & 809 (14.6) & 4,500 & 404 (7.3) \\
\hline Bai & 6,106 & $163(2.7)$ & 177 (2.9) & 5,863 & $97(1.6)$ \\
\hline Tibetan & 6,701 & $133(2.0)$ & $148(2.2)$ & 6,495 & $75(1.1)$ \\
\hline
\end{tabular}

of smoking were also grouped into three categories using the same methods. A trend test was conducted by entering the categorical variables as continuous parameters in the models. All analyses were conducted using R software, version 3.6.3 (R Core Team). The threshold for statistical significance was $P \leq 0.05$ based on 2 -sided tests.

\section{RESULTS}

Overall, $2.9 \%$ of participants reported having comorbid depressive and anxiety symptoms, $2.1 \%$ among men and $3.5 \%$ among women (Table 1). Among 4,947 (5.3\%) participants with depressive symptoms, $55.0 \%$ reported having anxiety symptoms, 


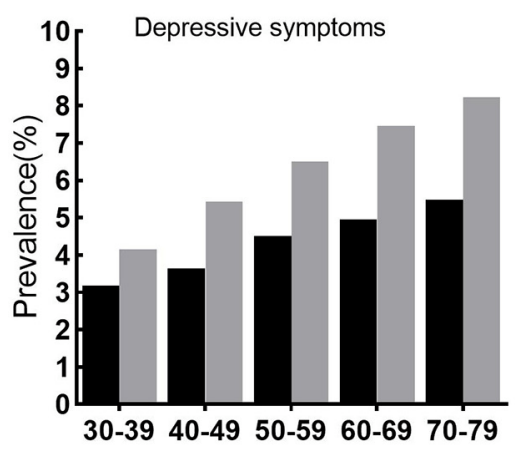

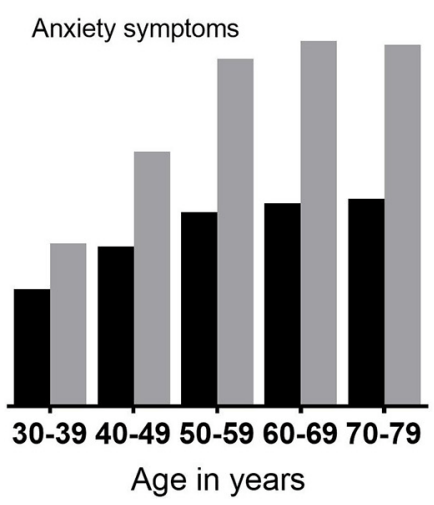

Comorbid depressive and anxiety symptoms
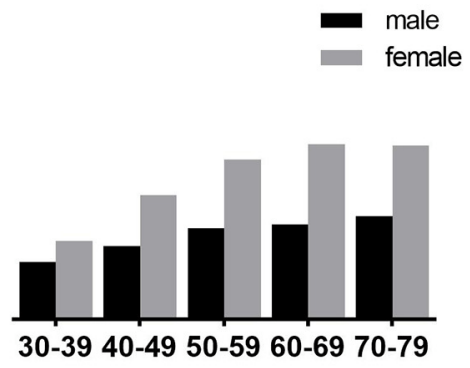

$30-39$ 40-49 50-59 60-69 70-79

FIGURE 1 | Prevalence of depressive symptoms and anxiety symptoms and comorbidity by age and sex.

while in 5,800 (6.2\%) participants with anxiety symptoms, $46.9 \%$ reported having depressive symptoms. Those participants with depressive symptoms had a 27 -fold elevated risk of anxiety symptoms compared with participants with no depressive symptoms (Supplemental Figure 1). For both men and women, the prevalence of comorbid depressive and anxiety symptoms increased until $\sim 60$ years $(P$ trend $<0.001)$; after 70 years, it was almost the same (Figure 1). Compared with participants who were neither depressive symptoms nor anxiety symptoms, those with comorbid depressive and anxiety symptoms were more likely to have poor education, lower household income, and be widowed/divorced (Table 1).

Among the seven ethnic groups, Tibetans had the lowest level of comorbid depressive and anxiety symptoms at $1.1 \%$. The Miao ethnic group had the highest levels of comorbidity $(7.3 \%)$ (Table 1). Compared with the Han ethnic group in Figure 2, the Tibetan ethnic group had a lower risk of comorbidity $(\mathrm{OR}=$ $0.38,95 \%$ CI: $0.29-0.50$ ), and the low-risk result was also found among the Bai ethnic group ( $\mathrm{OR}=0.50,95 \% \mathrm{CI}: 0.40-0.63)$ after adjusting for age, sex, education, family income, and lifestyles. The Miao ethnic group had an increased risk of comorbidity (OR $=3.04$, 95\% CI: 2.65-3.48).

The associations between lifestyle factors and comorbid depressive and anxiety symptoms are presented in Table 2. Medium and high physical activity was inversely related to comorbidities, with ORs of 0.69 (95\% CI: 0.62-0.76) and 0.82 (95\% CI: 0.74-0.91), respectively. Frequent alcohol consumption was found to be inversely associated with comorbid depressive and anxiety symptoms; this was observed in most ethnic groups and men, but the opposite was found in women (Supplemental Table 1). We did not find that the frequency of tea consumption was associated with comorbid depressive and anxiety symptoms. We also did not find an association between smoking or BMI and comorbidity.

Table 3 shows the adjusted ORs for comorbid depressive and anxiety symptoms associated with 20 common chronic diseases by adjusting for sociodemographic characteristics and lifestyle habits. We estimated the associations of depressive symptoms,

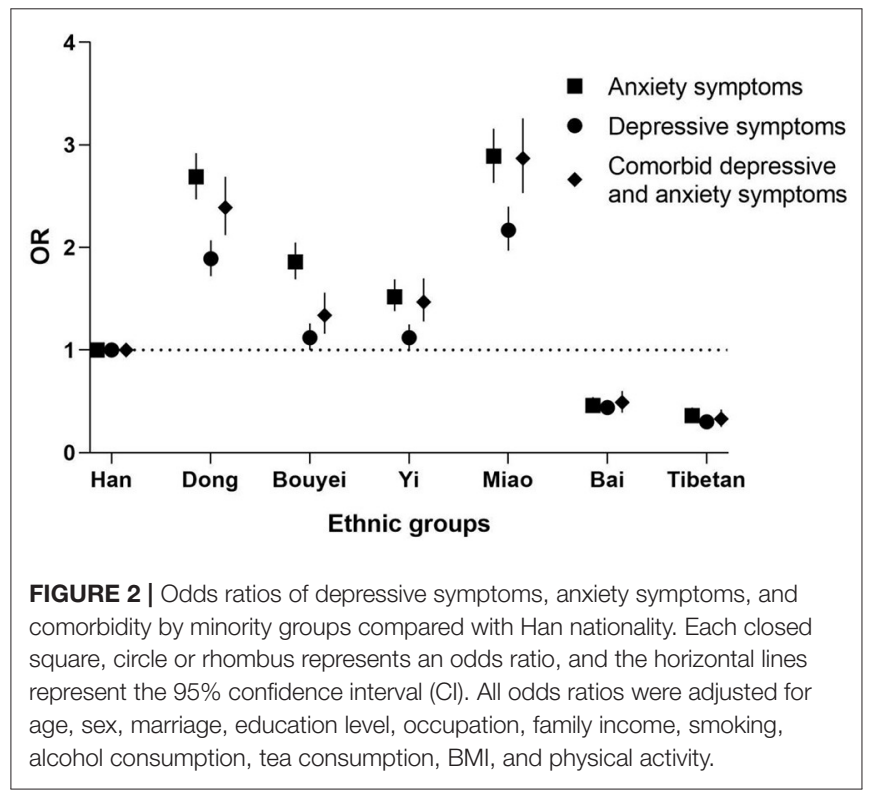

anxiety symptoms and comorbidities with each of these diseases separately. Individuals who reported having rheumatic heart disease were more likely to have comorbid depressive and anxiety symptoms with OR 6.25 (95\%CI: 4.06-9.62); the other diseases also strongly related with the comorbidity were pulmonary heart disease with OR 3.56 (95\%CI: 2.58-4.90), coronary heart disease with OR 2.94 (95\%CI: 2.48-3.48) and stroke with OR 2.78 (95\%CI: 2.04-3.79).

Compared with those who were excellent self-rated health status, the adjusted ORs of comorbid depressive and anxiety symptoms were increased with the reduced degree of self-rated health status, the ORs of the comorbidity were $2.81(95 \% \mathrm{CI}$ : 2.20-3.59) for "fair," 9.26 (95\%CI: 7.20-11.92) for "poor", and 33.60 (95\%CI: 25.16-44.87) for "very poor," with dose-response relationship (trend $P<0.001$ ). Similarly, compared with those 
TABLE 2 | Associations of lifestyles and behaviors with comorbid depressive and anxiety symptoms.

\begin{tabular}{|c|c|c|c|c|c|c|c|}
\hline \multirow[t]{2}{*}{ Variables* } & \multirow[b]{2}{*}{$\begin{array}{c}\text { Total }(N= \\
93,078)\end{array}$} & \multicolumn{2}{|c|}{ Depressive symptoms } & \multicolumn{2}{|c|}{ Anxiety symptoms } & \multicolumn{2}{|c|}{ Comorbid depressive and anxiety symptoms } \\
\hline & & Yes $(n=4,947)$ & $\begin{array}{l}\text { Odds ratio } \\
(95 \% \mathrm{Cl})\end{array}$ & Yes $(n=5,800)$ & $\begin{array}{l}\text { Odds ratio } \\
(95 \% \mathrm{Cl})\end{array}$ & Yes $(n=2,721)$ & $\begin{array}{l}\text { Odds ratio } \\
(95 \% \mathrm{Cl})\end{array}$ \\
\hline \multicolumn{8}{|l|}{ Smoking } \\
\hline No & 68,837 & 3,901 & 1 & 4,701 & 1 & 2,222 & 1 \\
\hline Past & 4,700 & 213 & $1.11(0.94,1.30)$ & 243 & $1.18(1.01,1.38)$ & 113 & $1.15(0.92,1.43)$ \\
\hline Current & 19,541 & 833 & $1.00(0.90,1.11)$ & 856 & $0.95(0.86,1.06)$ & 386 & $0.87(0.75,1.01)$ \\
\hline \multicolumn{8}{|c|}{$\begin{array}{l}\text { Duration of smoking } \\
\text { (years) }\end{array}$} \\
\hline$<24$ & 5,934 & 232 & $1.12(0.96,1.31)$ & 228 & $1.09(0.93,1.27)$ & 107 & $1.03(0.82,1.29)$ \\
\hline $24-42$ & 12,637 & 516 & $0.97(0.86,1.09)$ & 586 & $1.01(0.90,1.14)$ & 246 & $0.90(0.76,1.07)$ \\
\hline$\geq 42$ & 5,610 & 294 & $1.04(0.90,1.21)$ & 281 & $0.89(0.76,1.03)$ & 144 & $0.87(0.71,1.07)$ \\
\hline \multicolumn{8}{|c|}{ Alcohol consumption } \\
\hline No & 52,079 & 3,067 & 1 & 3,611 & 1 & 1,748 & 1 \\
\hline Sometime & 28,411 & 1,380 & $0.94(0.87,1.01)$ & 1,586 & $0.96(0.90,1.03)$ & 732 & $0.92(0.84,1.01)$ \\
\hline Frequently & 12,588 & 500 & $0.76(0.69,0.85)$ & 603 & $0.88(0.80,0.97)$ & 241 & $0.71(0.61,0.82)$ \\
\hline \multicolumn{8}{|c|}{$\begin{array}{l}\text { Frequency of alcohol } \\
\text { consumption }\end{array}$} \\
\hline $1-2 \mathrm{~d} /$ week & 3,055 & 136 & $0.92(0.77,1.11)$ & 159 & $1.04(0.87,1.24)$ & 67 & $0.87(0.67,1.13)$ \\
\hline $3-5 \mathrm{~d} /$ week & 2,557 & 89 & $0.69(0.56,0.87)$ & 119 & $0.92(0.75,1.12)$ & 45 & $0.69(0.51,0.94)$ \\
\hline $6-7 \mathrm{~d} /$ week & 6,972 & 275 & $0.67(0.59,0.78)$ & 324 & $0.78(0.69,0.89)$ & 129 & $0.61(0.50,0.74)$ \\
\hline \multicolumn{8}{|c|}{ Tea consumption } \\
\hline No & 63,106 & 3,774 & 1 & 4,594 & 1 & 2,135 & 1 \\
\hline Yes & 29,969 & 1,172 & $0.98(0.90,1.05)$ & 1,206 & $0.92(0.85,0.99)$ & 586 & $0.95(0.85,1.05)$ \\
\hline \multicolumn{8}{|c|}{$\begin{array}{l}\text { Frequency of tea } \\
\text { consumption }\end{array}$} \\
\hline 1-2 d/week & 3,096 & 140 & $0.85(0.71,1.02)$ & 144 & $0.96(0.81,1.13)$ & 68 & $0.96(0.75,1.24)$ \\
\hline 3-5 d/week & 3,862 & 131 & $0.98(0.90,1.06)$ & 149 & $0.95(0.80,1.12)$ & 64 & $0.87(0.68,1.13)$ \\
\hline 6-7 d/week & 22,251 & 854 & $1.03(0.86,1.22)$ & 858 & $0.88(0.81,0.96)$ & 426 & $0.93(0.83,1.05)$ \\
\hline \multicolumn{8}{|c|}{$\begin{array}{l}\text { Duration of tea } \\
\text { consumption (years) }\end{array}$} \\
\hline$<15$ & 7,061 & 343 & $1.09(0.97,1.22)$ & 351 & $1.00(0.89,1.12)$ & 174 & $1.07(0.91,1.25)$ \\
\hline $15-39$ & 15,513 & 564 & $0.94(0.85,1.04)$ & 586 & $0.92(0.83,1.01)$ & 280 & $0.92(0.80,1.05)$ \\
\hline$\geq 39$ & 7,247 & 252 & $0.87(0.75,1.00)$ & 254 & $0.76(0.66,0.88)$ & 124 & $0.78(0.64,0.96)$ \\
\hline \multicolumn{8}{|l|}{ BMI } \\
\hline$<18.5$ & 2,954 & 211 & $1.18(1.01,1.37)$ & 222 & $1.04(0.89,1.20)$ & 114 & $1.13(0.92,1.38)$ \\
\hline $18.5-23.9$ & 41,127 & 2,226 & 1 & 2,553 & 1 & 1,214 & 1 \\
\hline
\end{tabular}




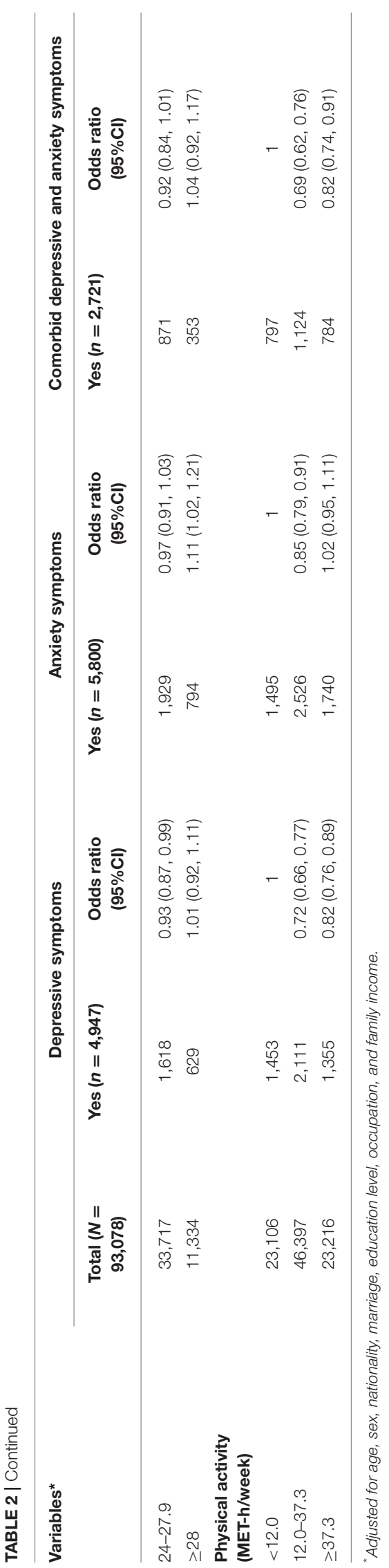

with a very satisfying life, the adjusted ORs of comorbid depressive and anxiety symptoms increased with a reduced degree of self-reported life satisfaction, with ORs of 2.76 ( $95 \% \mathrm{CI}$ : 2.44-3.14), 8.92 (95\% CI: 7.62-10.44), and 33.30 (95\% CI: 23.7346.74) for "neither satisfied nor dissatisfied," "unsatisfied," and "very unsatisfied," respectively, with a dose-response relationship (trend $P<0.001$ ) (Figure 3).

Individuals who reported having sleep disorders, a history of doctor-diagnosed psychiatric disorder or neurasthenia had a higher likelihood of having comorbid depressive and anxiety symptoms; the ORs of these mental disorders associated with comorbidities were 4.10 (95\% CI: 3.73-4.50), 10.09 (95\% CI: 7.54-13.49), and 5.38 (95\% CI: 4.41-6.56), respectively. The adjusted ORs increase progressively with the number of stressful life events experienced during the previous two years, with a dose-response relationship (trend $P<0.001$ ) (Figure 3).

Moreover, the associations between all of the above factors and comorbid depressive and anxiety symptoms were higher than that of depressive symptoms alone or anxiety symptoms alone (Table 3; Supplemental Figures 1, 2).

\section{DISCUSSION}

In this large multiethnic population-based study with seven ethnic groups aged 30-79 years from 5 provinces in Southwest China, $2.9 \%$ of participants reported having comorbid depressive and anxiety symptoms. Comparing the prevalence of $4.2-$ $12 \%$ of comorbid depressive and anxiety symptoms with a few previous studies, the total prevalence in our study was low $(25,26)$. The low prevalence could be underestimated because of the lack of mental health literacy and stigmainduced conscious non-disclosure reporting in the low-middle developing areas.

In the study, higher risks of comorbid depressive and anxiety symptoms were associated with female sex and low socioeconomic status, including poorer education, lower household income, and being widowed; the correlates were similar to depressive symptoms alone or anxiety symptoms alone. Surprisingly, frequent alcohol consumption was associated with lower risks of comorbid depressive and anxiety symptoms. These results were somewhat consistent with previous studies which demonstrated that moderate not heavy alcohol consumption had lower risk to have depression $(27,28)$. In this study, the rate of frequently drinking alcohol was $13.5 \%$ in total, $29.5 \%$ among men and $2.9 \%$ among women. A high frequency of drinking was almost 10 times more common in men than in women (Supplementary Table 1). Drinking alcohol, generally regarded as moderate drinking, is often combined with local culture and used to offer sacrifices and entertain guests in some ethnic groups. Drinking with other people is part of daily life entertainment among men from some ethnic groups. Therefore, it is not the alcohol itself but the activities of drinking, which might enable men to participate in social life and obtain social support, thereby reducing depressive 
TABLE 3 | The adjusted ORs of chronic diseases and comorbid depressive and anxiety symptoms.

\begin{tabular}{|c|c|c|c|c|c|c|c|}
\hline \multirow[t]{2}{*}{ Type of disease ${ }^{*}$} & \multirow[b]{2}{*}{$\begin{array}{c}\text { Total } \\
(N=93,078)\end{array}$} & \multicolumn{2}{|c|}{ Depressive symptoms } & \multicolumn{2}{|c|}{ Anxiety symptoms } & \multicolumn{2}{|c|}{ Comorbid depressive and anxiety symptoms } \\
\hline & & $\begin{array}{l}\text { With disease } \\
(n=4,947)\end{array}$ & $\begin{array}{l}\text { Odds ratio } \\
(95 \% \mathrm{Cl})\end{array}$ & $\begin{array}{l}\text { With disease } \\
(n=5,800)\end{array}$ & $\begin{array}{l}\text { Odds ratio } \\
(95 \% \mathrm{Cl})\end{array}$ & $\begin{array}{l}\text { With disease } \\
(n=2,721)\end{array}$ & $\begin{array}{l}\text { Odds ratio } \\
(95 \% \mathrm{Cl})\end{array}$ \\
\hline \multicolumn{8}{|l|}{ Diseases } \\
\hline No & 38,517 & 1,412 & 1 & 1,757 & 1 & 760 & 1 \\
\hline yes & 54,560 & 3,541 & $1.92(1.8,2.05)$ & 4,049 & $1.78(1.67,1.89)$ & 1,961 & $2.04(1.87,2.23)$ \\
\hline Hypertension & 16,063 & 1,124 & $1.34(1.25,1.45)$ & 1,293 & $1.27(1.18,1.37)$ & 632 & $1.38(1.25,1.52)$ \\
\hline Diabetes & 4,503 & 347 & $1.54(1.37,1.73)$ & 373 & $1.47(1.31,1.66)$ & 191 & $1.65(1.41,1.93)$ \\
\hline Hyperlipidemia & 7,366 & 468 & $1.72(1.55,1.91)$ & 498 & $1.68(1.52,1.86)$ & 259 & $1.93(1.68,2.22)$ \\
\hline Coronary heart disease & 2,478 & 309 & $2.57(2.25,2.92)$ & 342 & $2.52(2.22,2.86)$ & 178 & $2.94(2.48,3.48)$ \\
\hline Stroke & 675 & 80 & $2.41(1.89,3.08)$ & 81 & $2.20(1.72,2.81)$ & 48 & $2.78(2.04,3.79)$ \\
\hline Rheumatic heart disease & 212 & 40 & $4.70(3.28,6.75)$ & 39 & $4.08(2.83,5.87)$ & 27 & $6.25(4.06,9.62)$ \\
\hline Pulmonary heart disease & 398 & 80 & $3.21(2.47,4.17)$ & 79 & $2.48(1.90,3.24)$ & 51 & $3.56(2.58,4.90)$ \\
\hline Tuberculosis & 1,478 & 121 & $1.58(1.30,1.92)$ & 138 & $1.67(1.38,2.01)$ & 74 & $1.84(1.44,2.36)$ \\
\hline Chronic bronchitis & 5,794 & 568 & $1.93(1.75,2.12)$ & 618 & $1.80(1.64,1.98)$ & 328 & $2.12(1.87,2.40)$ \\
\hline Asthma & 1,320 & 154 & $2.29(1.92,2.73)$ & 154 & $2.06(1.72,2.46)$ & 81 & $2.37(1.87,3.01)$ \\
\hline Hepatitis & 2,589 & 200 & $1.95(1.67,2.27)$ & 190 & $1.72(1.47,2.01)$ & 99 & $1.94(1.57,2.39)$ \\
\hline Peptic ulcer & 2,699 & 232 & $1.98(1.72,2.28)$ & 247 & $1.92(1.67,2.21)$ & 136 & $2.33(1.94,2.79)$ \\
\hline Gastroenteritis & 11,687 & 974 & $1.94(1.80,2.09)$ & 1,049 & $1.79(1.66,1.92)$ & 563 & $2.15(1.95,2.37)$ \\
\hline Gallstone & 11,130 & 700 & $1.36(1.25,1.49)$ & 786 & $1.31(1.21,1.42)$ & 394 & $1.43(1.28,1.61)$ \\
\hline Fracture & 6,900 & 463 & $1.45(1.31,1.61)$ & 528 & $1.50(1.36,1.65)$ & 248 & $1.51(1.32,1.74)$ \\
\hline Rheumatic arthritis & 6,589 & 608 & $1.76(1.60,1.93)$ & 728 & $1.80(1.65,1.96)$ & 379 & $2.04(1.81,2.30)$ \\
\hline Rheumatoid arthritis & 2,556 & 281 & $2.24(1.96,2.56)$ & 347 & $2.41(2.13,2.72)$ & 186 & $2.80(2.38,3.30)$ \\
\hline Intervertebral disc disease & 16,710 & 1,343 & $1.68(1.57,1.80)$ & 1,578 & $1.67(1.57,1.78)$ & 785 & $1.87(1.71,2.04)$ \\
\hline Cerebral trauma & 1,900 & 183 & $1.94(1.66,2.28)$ & 212 & $2.03(1.74,2.36)$ & 99 & $2.10(1.69,2.60)$ \\
\hline Cancer & 780 & 66 & $1.54(1.19,1.99)$ & 68 & $1.33(1.02,1.72)$ & 38 & $1.62(1.16,2.27)$ \\
\hline
\end{tabular}

"Adjusted for age, sex, nationality, marriage, education level, occupation, family income, smoking, alcohol consumption, tea consumption, BMI, and physical activity. 


\begin{tabular}{|c|c|c|c|c|}
\hline Subgroup & $\begin{array}{l}\text { Comorbid depressive } \\
\text { and anxiety symptoms } \\
\qquad(\mathrm{N}=2721)\end{array}$ & $\begin{array}{c}\text { None } \\
(\mathrm{N}=85052)\end{array}$ & & $\begin{array}{l}\text { Odds Ratio }(95 \% \mathrm{Cl}) \\
\text { for comorbid depressive } \\
\text { and anxiety symptoms }\end{array}$ \\
\hline \multicolumn{5}{|l|}{ Satisfaction levels of life } \\
\hline Very satisfied & 421 & 26288 & 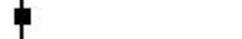 & Reference \\
\hline Satisfied & 982 & 41415 & & $1.47(1.31,1.66)$ \\
\hline Neither satisfied nor dissatisfied & 808 & 15420 & a & $2.76(2.44,3.14)$ \\
\hline Unsatisfied & 429 & 1833 & 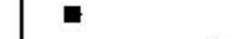 & $8.92(7.62,10.44)$ \\
\hline Very unsatisfied & 81 & 96 & & $33.3(23.73,46.74)$ \\
\hline \multicolumn{5}{|l|}{ Self-rated health status } \\
\hline Excellent & 70 & 8652 & & Reference \\
\hline Good & 331 & 33446 & & $1.12(0.87,1.46)$ \\
\hline Fair & 1129 & 35864 & $\square$ & $2.81(2.20,3.59)$ \\
\hline Poor & 919 & 6571 & + & $9.26(7.20,11.92)$ \\
\hline Very poor & 269 & 480 & 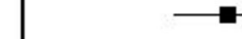 & $33.6(25.16,44.87)$ \\
\hline \multicolumn{5}{|l|}{ Number of stressful life events } \\
\hline 0 & 1215 & 62209 & & Reference \\
\hline 1 & 809 & 17043 & C & $2.17(1.98,2.38)$ \\
\hline 2 & 393 & 4262 & $\mathbf{a}$ & $3.83(3.38,4.33)$ \\
\hline $3+$ & 295 & 1492 & $=$ & $8.43(7.27,9.77)$ \\
\hline \multicolumn{5}{|l|}{ Sleep disorder } \\
\hline No & 616 & 49870 & 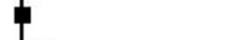 & Reference \\
\hline Yes & 2105 & 35180 & $\mathbf{a}$ & $4.10(3.73,4.50)$ \\
\hline \multicolumn{5}{|l|}{ Psychiatric disorder } \\
\hline No & 2652 & 84818 & & Reference \\
\hline Yes & 69 & 233 & - & $10.09(7.54,13.49)$ \\
\hline \multicolumn{5}{|l|}{ Neurasthenis } \\
\hline No & 2590 & 84158 & & Reference \\
\hline \multirow[t]{2}{*}{ Yes } & 131 & 893 & ${ }_{11} \mathbf{n}_{1}$ & $5.38(4.41,6.56)$ \\
\hline & & & $\begin{array}{lllll}0 & 5 & 10 & 20 \\
& & & & \text { OR }(95 \% \mathrm{Cl})\end{array}$ & 50 \\
\hline
\end{tabular}

FIGURE 3 | Adjusted odds ratios by health-related conditions and status for comorbid depressive and anxiety symptoms. Each closed square represents an odds ratio, and the horizontal lines represent the 95\% confidence interval (Cl). All odds ratios were adjusted for age, sex, nationality, marriage, education level, occupation, income, smoking, alcohol consumption, tea consumption, BMl, physical activity, and diseases.

symptoms and anxiety symptoms. Further gender-specific, the amount of alcohol consumption studies is needed to explore the associations of alcohol consumption with comorbid depressive and anxiety symptoms.

The Miao minority and Dong minority reported the highest risks of comorbid depressive and anxiety symptoms, followed by the Bouyei and Yi minorities. These ethnic minorities live in economically underdeveloped areas and were in the process of accelerating urbanization, which resulted in not only geographical environmental changes but also living modes, interpersonal communication modes, traditional values, and cultural changes $(29,30)$. Previous studies have shown that people from these urbanizing minority areas suffer from residential migration, lifestyle changes, and cultural adjustment and integration; compared with people already living in welldeveloped cities, these residents were more likely to have depression or anxiety $(13,14)$. The considerable differences in comorbid depressive and anxiety symptoms among ethnic groups could not only be explained entirely due to changes in the environment. There might also be differences in the interaction between genes and the environment, which requires more indepth research $(31,32)$.

In this study, we investigated the top 20 common chronic diseases and cancers in the southwest multiethnic populations. We found that all chronic diseases and cancers were associated with comorbid depressive and anxiety symptoms; the associations were stronger than depressive symptoms alone or anxiety symptoms alone. Some previous studies have demonstrated that stroke, coronary heart disease, and cancers are strongly associated with depressive symptoms or anxious symptoms (33-36). We confirmed these results. Moreover, we found rheumatic heart disease had the highest risk with comorbid depressive and anxiety symptoms among these 20 kinds of diseases. Comorbid depressive sand anxiety symptoms can increase patients' medical overuse and are associated with slower recovery, reducing adherence to treatment than depressive symptoms or anxiety symptoms alone (37). These results suggested that, based on the current situation of the severe shortage of mental health resources in southwest minority areas, policymakers should formulate policies to improve comorbid 
depressive and anxiety symptoms among patients with key chronic diseases in basic community health centers or rural township health centers.

Although a simple question was designed to measure life satisfaction and self-rated health status, we found strong linear associations. Compared with those who were very satisfied with life and excellent health status, participants who were very unsatisfied with life and very poor health status had 33-fold and 34-fold higher risks of comorbid depressive and anxiety symptoms, respectively. This suggests that primary health care institutions should pay special attention to residents who complain about their life status or physical conditions because they are likely to suffer from depressive symptoms, anxiety symptoms or both (9).

Stressful life events as strong risk factors for depressive symptoms and anxiety symptoms have been reported in populations of different ages. One psychological mechanism suggests that rumination mediates the longitudinal relationship between stressful life events and comorbid depressive and anxiety symptoms (38), while the genetic mechanism suggests that stressful life events may change mitochondrial DNA and telomere length to cause depression (39). Previous studies have demonstrated that depressive symptoms and anxiety symptoms are associated with sleep disorders, other psychiatric disorders and neurasthenia $(24,40)$. The present study showed that the associations between these mental problems and the comorbidity were stronger than those of depressive symptoms alone or anxiety symptoms alone. Due to culture, customs and discrimination against mental disorders, many ethnic minority residents may report neurasthenia or sleep disorders if they suffer from depressive symptoms or anxiety symptoms $(7,41)$. The results suggested that general medical practitioners from primary care institutions should be given adequate training to improve the ability to recognize psychological problems to better identify and manage depressive and anxiety symptoms.

\section{Strengths and Limitations}

Strengths of this study were the large sample size, the multiethnic population involved, and the use of internationally validated scales for assessing depressive symptoms and anxiety symptoms in economically underdeveloped areas. Moreover, the wide information collected included socioeconomic, lifestyles, physical and mental health status, other disorders, and chronic diseases. However, this study has some limitations. First, the study was cross-sectional in nature, so no causal inferences could be made. Second, the study population was enrolled voluntarily, and individuals who were suffering from severe depression or anxiety disorders were less likely to participate in this investigation, which might be another reason for the low prevalence. Third, due to the lack of information on cultural characteristics and differences in the urbanization process, the reasons for differences in depressive symptoms and anxiety symptoms among different multiple ethnic groups were not further explored. Fourth, we used the PHQ-2 and GAD-2 to measure depressive symptoms and anxiety symptoms. Although these two scales have been demonstrated to be suitable for application in community-based epidemically investigations and their reliability and validity were validated to be good for the present study population, overestimated or underestimated depressive symptoms and anxiety symptoms might still occur because of a lack of clinical professional psychiatric evaluations. Participants who were screened positive should be further evaluated with clinical diagnostic instruments or a direct interview with clinical psychologists to determine whether they met the criteria for both major depressive disorder and anxiety disorder.

\section{CONCLUSIONS}

In this large population-based study of 93,078 adults from multiple ethnic groups in Southwest China, the prevalence of comorbid depressive and anxiety symptoms considerably varies among multiple ethnic groups. Stressful life events, physical and mental well-being and certain health conditions are positively associated with comorbid depressive and anxiety symptoms while the high frequency of physical activity and male alcohol consumption has negative associations. All the associations are stronger than depressive symptoms alone and anxiety symptoms alone. Since medical resources are relatively scarce in southwest multiethnic areas, these findings are considered valuable for policymakers to develop prevention programs in early identifying and intervening in the vulnerable populations with comorbid depressive and anxiety symptoms.

\section{DATA AVAILABILITY STATEMENT}

The original contributions presented in the study are included in the article/Supplementary Material, further inquiries can be directed to the corresponding authors.

\section{ETHICS STATEMENT}

The studies involving human participants were reviewed and approved by Sichuan University Medical Ethical Review Board (K2016038). The patients/participants provided their written informed consent to participate in this study.

\section{AUTHOR CONTRIBUTIONS}

XZ, PL, and QL contributed to the study conception and design. QL and PW contributed to analyzed data and draft the first version of manuscript. $\mathrm{YB}, \mathrm{SW}, \mathrm{ZS}$, and JZ contributed to collected and clear up data. HS, YL, and XL contributed to critically revised manuscript. All authors read and approved the final manuscript.

\section{FUNDING}

This study was supported by the National Key R\&D Program 'Precision Medicine Initiative' of China (Grants 2017YFC0907303 and 2017YFC0907302) and the Sichuan Science and Technology Program (Grants 2020JDJQ0014 and 2020YFS0216). The funding had no role in the design, analysis or writing of this manuscript. 


\section{ACKNOWLEDGMENTS}

The authors would like to thank all the participants in this study, as well as thank all the interviewers and members of the survey teams in each of the study sites and communities.

\section{REFERENCES}

1. Braam AW, Copeland JR, Delespaul PA, Beekman AT, Como A, Dewey M, et al. Depression, subthreshold depression and comorbid anxiety symptoms in older Europeans: results from the EURODEP concerted action. J Affect Disord. (2014) 155:266-72. doi: 10.1016/j.jad.2013.11.011

2. Lamers F, van Oppen P, Comijs HC, Smit JH, Spinhoven P, van Balkom AJ, et al. Comorbidity patterns of anxiety and depressive disorders in a large cohort study: the Netherlands Study of Depression and Anxiety (NESDA). J Clin Psychiatry. (2011) 72:341-8. doi: 10.4088/JCP.10m06176blu

3. Collaborators GDaIIaP. Global, regional, and national incidence, prevalence, and years lived with disability for 354 diseases and injuries for 195 countries and territories, 1990-2017: a systematic analysis for the Global Burden of Disease Study 2017. Lancet. (2018) 392:1789-858. doi: 10.1016/S0140-6736(17)32154-2

4. Baxter AJ, Scott KM, Vos T, Whiteford HA. Global prevalence of anxiety disorders: a systematic review and meta-regression. Psychol Med. (2013) 43:897-910. doi: 10.1017/S003329171200147X

5. Whiteford HA, Degenhardt L, Rehm J, Baxter AJ, Ferrari AJ, Erskine HE, et al. Global burden of disease attributable to mental and substance use disorders: findings from the Global Burden of Disease Study 2010. Lancet. (2013) 382:1575-86. doi: 10.1016/S0140-6736(13)61611-6

6. Ferrari AJ, Somerville AJ, Baxter AJ, Norman R, Patten SB, Vos T, et al. Global variation in the prevalence and incidence of major depressive disorder: a systematic review of the epidemiological literature. Psychol Med. (2013) 43:471-81. doi: 10.1017/S0033291712001511

7. Wu YT, Kralj C, Acosta D, Guerra M, Huang Y, Jotheeswaran AT, et al. The association between, depression, anxiety, and mortality in older people across eight low- and middle-income countries: results from the 10/66 cohort study. Int J Geriatr Psychiatry. (2020) 35:29-36. doi: 10.1002/gps.5211

8. Belzer K, Schneier FR. Comorbidity of anxiety and depressive disorders: issues in conceptualization, assessment, and treatment. J Psychiatr Pract. (2004) 10:296-306. doi: 10.1097/00131746-200409000-00003

9. Hirschfeld RM. The comorbidity of major depression and anxiety disorders: recognition and management in primary care. Prim Care Companion J Clin Psychiatry. (2001) 3:244-54. doi: 10.4088/PCC.v0 3n0609

10. Chisholm D, Sweeny K, Sheehan P, Rasmussen B, Smit F, Cuijpers $\mathrm{P}$, et al. Scaling-up treatment of depression and anxiety: a global return on investment analysis. Lancet Psychiatry. (2016) 3:415-24. doi: 10.1016/S2215-0366(16)30024-4

11. Cyranowski JM, Schott LL, Kravitz HM, Brown C, Thurston RC, Joffe H, et al. Psychosocial features associated with lifetime comorbidity of major depression and anxiety disorders among a community sample of mid-life women: the SWAN mental health study. Depress Anxiety. (2012) 29:10507. doi: $10.1002 /$ da. 21990

12. Zhao W, Zhang Y, Liu X, Yue J, Hou L, Xia X, et al. Comorbid depressive and anxiety symptoms and frailty among older adults: findings from the West China health and aging trend study. J Affect Disord. (2020) 277:9706. doi: 10.1016/j.jad.2020.08.070

13. Chen J, Chen S, Landry PF. Migration, environmental hazards, and health outcomes in China. Social Sci Med. (2013) 80:8595. doi: 10.1016/j.socscimed.2012.12.002

14. Harpham T. Urbanization and mental health in developing countries: a research role for social scientists, public health professionals and social psychiatrists. Social Sci Med. (1994) 39:233-45. doi: 10.1016/0277-9536(94)90332-8

\section{SUPPLEMENTARY MATERIAL}

The Supplementary Material for this article can be found online at: https://www.frontiersin.org/articles/10.3389/fpubh. 2021.783687/full\#supplementary-material

15. Wang R, Xue D, Liu Y, Chen H, Qiu Y. The relationship between urbanization and depression in China: the mediating role of neighborhood social capital. Int J Equity Health. (2018) 17:105. doi: 10.1186/s12939-018-0825-X

16. Zhao X, Hong F, Yin J, Tang W, Zhang G, Liang X, et al. Cohort Profile: the China Multi-Ethnic Cohort (CMEC) study. Int J Epidemiol. (2021) 50:7217211. doi: 10.1093/ije/dyaa185

17. Kroenke K, Spitzer RL, Williams JB. The patient health questionnaire2: validity of a two-item depression screener. Med Care. (2003) 41:128492. doi: 10.1097/01.MLR.0000093487.78664.3C

18. Kroenke K, Spitzer RL, Williams JB, Monahan PO, Löwe B. Anxiety disorders in primary care: prevalence, impairment, comorbidity, and detection. Ann Intern Med. (2007) 146:31725. doi: 10.7326/0003-4819-146-5-200703060-00004

19. Löwe B, Kroenke K, Gräfe K. Detecting and monitoring depression with a two-item questionnaire (PHQ-2). J Psychosom Res. (2005) 58:16371. doi: 10.1016/j.jpsychores.2004.09.006

20. Luo Z, Li Y, Hou Y, Liu X, Jiang J, Wang Y, et al. Gender-specific prevalence and associated factors of major depressive disorder and generalized anxiety disorder in a Chinese rural population: the Henan rural cohort study. BMC Public Health. (2019) 19:1744. doi: 10.1186/s12889-019-8086-1

21. Luo Z, Li Y, Hou Y, Zhang H, Liu X, Qian X, et al. Adaptation of the twoitem generalized anxiety disorder scale (GAD-2) to Chinese rural population: a validation study and meta-analysis. Gen Hosp Psychiatry. (2019) 60:506. doi: 10.1016/j.genhosppsych.2019.07.008

22. Levis B, Benedetti A, Thombs BD. Accuracy of Patient Health Questionnaire-9 (PHQ-9) for screening to detect major depression: individual participant data meta-analysis. BMJ. (2019) 365:11476. doi: 10.1136/bmj.11476

23. Sun X, Zheng B, Lv J, Guo Y, Bian Z, Yang L. et al. Sleep behavior and depression: findings from the China Kadoorie Biobank of 05 million Chinese adults. J Affect Disord. (2018) 229:120-4. doi: 10.1016/j.jad.2017.12.058

24. Chen Y, Bennett D, Clarke R, Guo Y, Yu C, Bian Z. et al. Patterns and correlates of major depression in Chinese adults: a cross-sectional study of 05 million men and women. Psychol Med. (2017) 47:95870. doi: 10.1017/S0033291716002889

25. Blay SL, Fillenbaum GG, Mello MF, Quintana MI, Mari JJ, Bressan RA, et al. 12-month prevalence and concomitants of DSM-IV depression and anxiety disorders in two violence-prone cities in Brazil. J Affect Disord. (2018) 232:204-11. doi: 10.1016/j.jad.2018.02.023

26. Moffitt TE, Harrington H, Caspi A, Kim-Cohen J, Goldberg D, Gregory AM, et al. Depression and generalized anxiety disorder: cumulative and sequential comorbidity in a birth cohort followed prospectively to age 32 years. Arch Gen Psychiatry. (2007) 64:651-60. doi: 10.1001/archpsyc.64.6.651

27. Gémes K, Forsell Y, Janszky I, László KD, Lundin A, Ponce De Leon A, et al. Moderate alcohol consumption and depression - a longitudinal population-based study in Sweden. Acta Psychiatr Scand. (2019) 139:52635. doi: 10.1111/acps.13034

28. Noh JW, Juon HS, Lee S, Kwon YD. Atypical epidemiologic finding in association between depression and alcohol use or smoking in Korean male: Korean longitudinal study of aging. Psychiatry Investig. (2014) 11:27280. doi: 10.4306/pi.2014.11.3.272

29. Liu F WW. Adaptation and transformation of the Miao culture. J Guizhou Univ. (2015) 33:107-10. doi: 10.15958/j.cnki.gdxbshb.2015.01.018

30. K S. Study on the Change of Life Style of The Buyi and Miao ethnic groups in Qiannan Prefecture under the background of urbanization. Heihe J. (2016) 227:185-8. doi: 10.14054/j.cnki.cn23-1120/c.2016.05.079

31. Lacerda-Pinheiro SF, Pinheiro Junior RF, Pereira de Lima MA, Lima da Silva CG, Vieira dos Santos Mdo S, Teixeira Júnior AG, et al. Are there depression 
and anxiety genetic markers and mutations? A systematic review. J Affect Disord. (2014) 168:387-98. doi: 10.1016/j.jad.2014.07.016

32. Smoller JW. The genetics of stress-related disorders: PTSD, depression, and anxiety disorders. Neuropsychopharmacology. (2016) 41:297-319. doi: 10.1038/npp.2015.266

33. DeJean D, Giacomini M, Vanstone M, Brundisini F. Patient experiences of depression and anxiety with chronic disease: a systematic review and qualitative meta-synthesis. Ont Health Technol Assess Ser. (2013) 13:1-33. https://pubmed.ncbi.nlm.nih.gov/24228079

34. Broomfield NM, Quinn TJ, Abdul-Rahim AH, Walters MR, Evans JJ. Depression and anxiety symptoms post-stroke/TIA: prevalence and associations in cross-sectional data from a regional stroke registry. $B M C$ Neurol. (2014) 14:198. doi: 10.1186/s12883-014-0198-8

35. Niedzwiedz CL, Knifton L, Robb KA, Katikireddi SV, Smith DJ. Depression and anxiety among people living with and beyond cancer: a growing clinical and research priority. BMC Cancer. (2019) 19:943. doi: $10.1186 / \mathrm{s} 12885-019-6181-4$

36. Mitchell AJ, Ferguson DW, Gill J, Paul J, Symonds P. Depression and anxiety in long-term cancer survivors compared with spouses and healthy controls: a systematic review and meta-analysis. Lancet Oncol. (2013) 14:72132. doi: 10.1016/S1470-2045(13)70244-4

37. Deschênes SS, Burns RJ, Schmitz N. Associations between diabetes, major depressive disorder and generalized anxiety disorder comorbidity, and disability: findings from the 2012 Canadian Community Health Survey-Mental Health (CCHS-MH). J Psychosom Res. (2015) 78:13742. doi: 10.1016/j.jpsychores.2014.11.023

38. Ruscio AM, Gentes EL, Jones JD, Hallion LS, Coleman ES, Swendsen J. Rumination predicts heightened responding to stressful life events in major depressive disorder and generalized anxiety disorder. J Abnorm Psychol. (2015) 124:17-26. doi: 10.1037/abn0000025
39. Cai N, Chang S, Li Y, Li Q, Hu J, Liang J, et al. Molecular signatures of major depression. Curr Biol. (2015) 25:1146-56. doi: 10.1016/j.cub.2015. 10.065

40. Luik AI, Zuurbier LA, Direk N, Hofman A, Van Someren EJ, Tiemeier H. 24-hour activity rhythm and sleep disturbances in depression and anxiety: a population-based study of middle-aged and older persons. Depress Anxiety. (2015) 32:684-92. doi: 10.1002/da.22 355

41. Parker G, Gladstone G, Chee KT. Depression in the planet's largest ethnic group: the Chinese. Am J Psychiatry. (2001) 158:857-64. doi: 10.1176/appi.ajp.158.6.857

Conflict of Interest: The authors declare that the research was conducted in the absence of any commercial or financial relationships that could be construed as a potential conflict of interest.

Publisher's Note: All claims expressed in this article are solely those of the authors and do not necessarily represent those of their affiliated organizations, or those of the publisher, the editors and the reviewers. Any product that may be evaluated in this article, or claim that may be made by its manufacturer, is not guaranteed or endorsed by the publisher.

Copyright $\odot 2021$ Liu, Wangqing, Baima, Wang, Shen, Zhou, Song, Liu, Liu, Luo and Zhao. This is an open-access article distributed under the terms of the Creative Commons Attribution License (CC BY). The use, distribution or reproduction in other forums is permitted, provided the original author(s) and the copyright owner(s) are credited and that the original publication in this journal is cited, in accordance with accepted academic practice. No use, distribution or reproduction is permitted which does not comply with these terms. 